\title{
Cold Fusion Based on Matter-Antimatter Plasma Formed in Molecular Crystals
}

\author{
Mohamed Assaad Abdel-Raouf ${ }^{1 *}$, Abdelfattah T. Elgendy ${ }^{1}$, Amr Abd Al-Rahman Youssef ${ }^{2}$ \\ ${ }^{1}$ Physics Department, Faculty of Science, Ain Shams University, Cairo, Egypt \\ ${ }^{2}$ Mathematics Department, Faculty of Science, Al-Azhar University, Cairo, Egypt \\ Email: *assaad@sci.asu.edu.eg, amraay2003@gmail.com, abdelfattah.elgendy@sci.asu.edu.eg
}

How to cite this paper: Abdel-Raouf, M.A., Elgendy, A.T. and Youssef, A.A.A.-R. (2022) Cold Fusion Based on Matter-Antimatter Plasma Formed in Molecular Crystals. Journal of High Energy Physics, Gravitation and Cosmology, 8, 56-66.

https://doi.org/10.4236/jhepgc.2022.81004

Received: August 19, 2021

Accepted: November 23, 2021

Published: November 26, 2021

Copyright ( $\odot 2022$ by author(s) and Scientific Research Publishing Inc. This work is licensed under the Creative Commons Attribution International License (CC BY 4.0).

http://creativecommons.org/licenses/by/4.0/ (c) (i) Open Access

\begin{abstract}
The main purpose of this work is to shed light on the possibility of producing huge amount of energy based on the construction matter-antimatter plasma in a molecular crystal. It is assumed that two beams of isothermal hydrogen and antihydrogen are injected into a palladium crystal leading to a plasma state composed of particles and antiparticles. The collapse of this state releases a huge amount of energy which can be used as fuel for space shuttles. Thus, the novel system of isothermal pressure interaction enhances the energy power carried out by the quantum ion acoustic soliton (QIAS). In addition to the energy power released from the particle-antiparticle annihilation. The probability of merging the energy from these two cases is available at certain condition. The released energy may be a significant step in solving the energy scape of Tokomak to produce fusion energy. The study starting from the one-dimensional quantum hydrodynamic model (in which the term of electronpositron and proton-antiproton for hydrogen-antihydrogen is included), a Korteweg de Vries equation ( $\mathrm{kdv}$ ) is derived, the QIAS energy experiences and the annihilation energy power are calculated. It is found that the total energy of QIAS and the energy resulting from hydrogen-antihydrogen annihilation are important step towards the establishment of a cold fusion power station.
\end{abstract}

\section{Keywords}

Cold Fusion, Annihilation Energy, Plasma Energy, Ion-Acoustic Plasma, Cold and Hot Plasma, Matter-Antimatter Plasma in Molecular Crystals, Fuel for Space Shuttles in Palladium Crystals

\section{Introduction}

Antihydrogen, an atom composed of an antiproton orbited by a positron, was 
created at CERN in 1995 [1]. Fundamental development of the physics of particle-antiparticle interaction at low energy was achieved by the ATHENA GROUP at CERN in 2006 [2]. It was proved for the first time that the collision of a proton with an antihydrogen leads to the formation of a bound state composed of a proton and an antiproton, (identified by the Authors as antiprotonic hydrogen and known also as protonium), in which both particles are rotating around each other. The binding energy of the antiprotonic hydrogen was calculated as Es = $-0.75 \mathrm{keV}$ and the lifetime is $\tau \sim 1.0 \times 10^{-6} \mathrm{~s}$. Formation of protonium ions was theoretically confirmed by Abdel-Raouf [3]. Cold fusion based on matter-antimatter interaction was proposed and studied by Abdel-Raouf [4] [5] [6]. On the other hand, the last few years have shown a great deal of interest devoted mainly to the dynamics and applications of plasmas [7].

An interesting application will be announced in this article in which ion acoustic solitons (IAS) are formed in a compound plasma system of hydrogen and antihydrogen. The study of analog of IAS, using a simplified hydrodynamic model was previously achieved [8]. Since that, there has been a renewed interest in plasmas, covering different plasma modes, instabilities, and other nonlinear effects [9] [10] [11]. The most important quantities in our study are phase velocity, amplitude, and width, and the existence conditions of IAS. Considerable attention will be paid to the possible role; these quantities may play in the energy carried by the IAS. Let us recall that the interacting solitons may split, fuse, and exchange energies during their collisions. Many investigations have been made of the soliton energy for different plasma situations [11].

The large-scale oscillations are termed "solitons" [12]. IAS are nonlinear and coherent plasma structures that qualitatively share many properties with the lowfrequency fluctuations we have measured in the system of high energetic plasma of hydrogen-antihydrogen collision [13]. Solitons propagate in plasma near the ion acoustic speed, they are coherent and can be periodically excited. In a departure with previous linear perturbation analyses of electron-positron collision, we propose that the generation of IAS may be the source of coherent low frequency oscillations. Thus, IAS are coherent plasma oscillations born out of a balance between the nonlinearity of convection and wave dispersion. These waves have been studied extensively both experimentally and theoretically since the 1960s in double plasma devices [14] [15] [16]. To interpret these trends, we note that previous studies have shown that similar types of coherent structures propagating concurrently with ion acoustic solitons (IAS) energy. In the present work, we first review the relevant theory of IAS. We then interpret our proposed experimental measurements of hydrogen-antihydrogen collision through within the lines of this analytical theory to obtain the average value of the energy. On the other hand, in particle physics, annihilation is the process that occurs when a particle collides with its respective antiparticle to produce electromagnetic radiation, such as an electron colliding with a positron to produce two photons. High energetic electron-positron collision (more than $209 \mathrm{GeV}$ ) may lead to the pro- 
duction of particle-antiparticle pairs of larger masses, which is the basic concept of the LEP collider [16] [17] at CERN composed of a circular collider the circumference of which is $27 \mathrm{~km}$. The last decade has shown the establishment of the large hadron collider (LHC) based on high energetic proton-proton collisions occurring in a tunnel with 50-kilometer circumference [17] [18].

In our study, we will focus on the formation of electron-positron and protonantiproton plasma prior the annihilation process when two thermalized beams of hydrogen and antihydrogen are injected in a palladium crystal, (see Appendix A). The released energy will be calculated and added to the fusion energy of these two fundamental atoms. Apart from this introduction, the content of this paper is distributed in other three sections. The next section deals with the mathematical model employed for describing the plasma state. Section 3 is devoted to results and discussion of our calculation in addition to the anticipated hydrogen-antihydrogen fusion energy. In Section 4 we present the main conclusions drawn from our investigations. The paper ends with the complete list of references mentioned in the text as well as two Appendices A and B, the first of which deals with the main features of palladium crystals.

\section{Mathematical Model}

Solitons are known to exist in a dynamic equilibrium with ion-acoustic turbulence, which has been measured in the cathode plume [18]. The waveform for solitons can be derived from the $\sigma$ fluid equations for ions and electrons and Poisson equations. In the ion frame of reference, the ion continuity for an $\alpha$ particle is given by:

$$
\frac{\partial n_{\alpha}}{\partial t}+\frac{\partial}{\partial x}\left(n_{\alpha} u_{\alpha}\right)=0
$$

The equation of motion for $\alpha$-particle is given by:

$$
\frac{\partial u_{\alpha}}{\partial t}+u_{\alpha} \frac{\partial u_{\alpha}}{\partial x}+M_{\alpha} \sigma_{\alpha}\left(1 / n_{\alpha}\right) \frac{\partial n_{\alpha}}{\partial x}+Q_{\alpha} M_{\alpha} \frac{\partial \varphi}{\partial x}=0 .
$$

where, $\alpha=e$. For electron, $p$ for positron, $p r^{+}$for proton, and $p r^{-}$for antiproton.

Poisson equation is expressed as follow:

$$
\frac{\mathrm{d}^{2} \phi}{\mathrm{d} x^{2}}=\left(n_{p r^{-}}+n_{e}\right)-\left(n_{p}+n_{p r^{+}}\right) .
$$

where, $M_{\alpha}=\frac{m_{p r^{+}}}{m_{\alpha}}$, such that $m_{p r^{+}}=m_{p r^{-}}$and $m_{e}=m_{p}, Q_{e, p r^{-}}=-e$ and $Q_{p, p r^{+}}=e, \sigma_{\alpha}=\frac{T_{\alpha}}{T_{e}}$. Introducing the following dimensionless variables $x=\left(\frac{\omega_{p r}}{C_{s}}\right) x, \quad \tau=\omega_{p r} t, \phi=\left(\frac{e}{2 K_{B} T_{e}}\right) \phi, \quad C_{s}=\sqrt{\frac{2 K_{B} T_{e}}{m_{i}}}$ and the plasma frequency $\omega_{p r}=\sqrt{n_{0} e^{2} / m_{e} \varepsilon}$. 


\subsection{Linear Analysis}

The density $n_{\alpha}$, velocity $u_{\alpha}$ and potential $\phi$ of our novel system are introduced as:

$$
\begin{gathered}
n_{\alpha}=n_{\alpha 0}+\varepsilon n_{\alpha 1} \mathrm{e}^{i(k x-\omega t)}, \\
u_{\alpha}=u_{\alpha 0}+\varepsilon u_{\alpha 1} \mathrm{e}^{i(k x-\omega t)}, \\
\phi=\varepsilon \phi_{1} \mathrm{e}^{i(k x-\omega t)} .
\end{gathered}
$$

where, $n_{\alpha 0} \neq 0$ and $u_{\alpha 0} \neq 0$, to obtain the density and velocity of $\alpha$-particle which described in terms of the electrostatic potential $\phi_{1}$ as:

$$
\begin{gathered}
n_{\alpha 1}=\frac{Q_{\alpha} M_{\alpha} n_{\alpha 0} k^{2}}{\left(k u_{\alpha}^{0}-\omega\right)^{2}-M_{\alpha} \rho_{\alpha} k^{2}} \phi_{1}, \\
u_{\alpha 1}=\frac{k Q_{\alpha} M_{\alpha}\left(k u_{\alpha}^{0}-\omega\right)}{\left(k u_{\alpha}^{0}-\omega\right)^{2}-M_{\alpha} \rho_{\alpha} k^{2}} \phi_{1} .
\end{gathered}
$$

And the neutral condition for the initial densities which is taken in the form:

$$
n_{p r^{+} 0}+n_{p 0}=n_{p r-0}+n_{e 0} \text {. }
$$

And the dispersion relation between $\omega$ and $k$ which is given by:

$$
\frac{\Lambda n_{e 0}}{f_{e}}+\frac{\Lambda n_{p 0}}{f_{p}}+\frac{n_{p r^{+} 0}}{f_{p r^{+}}}+\frac{n_{p r^{-} 0}}{f_{p r^{-}}}=1 .
$$

where, $\Lambda=m_{p r^{+}} / m_{p e}$ is the relative mass between a proton mass and electron mass, $f_{e}=\left(k n_{e 0}-\omega\right)^{2}-\Lambda k^{2}, f_{p}=\left(k n_{p 0}-\omega\right)^{2}-\sigma_{p} \Lambda k^{2}$, $f_{p r^{+}}=\left(k n_{p r^{+} 0}-\omega\right)^{2}-\sigma_{p r^{+}} \Lambda k^{2}$, and $f_{p r^{-}}=\left(k n_{p r^{-} 0}-\omega\right)^{2}-\sigma_{p r^{-}} \Lambda k^{2}$ such as $\omega \neq 0$, and $k \neq 0$.

\subsection{Non-Linear Analysis}

In this section, stretching coordinates is introduced as: $\xi=\varepsilon^{\frac{1}{2}}(x-v t), \tau=\varepsilon^{\frac{3}{2}} v t$. The perturbations of density, velocity for article and electric potential are given by $n_{\alpha}=n_{\alpha}^{0}+\varepsilon n_{\alpha}^{(1)}+\varepsilon^{2} n_{\alpha}^{(2)}+\cdots, u_{\alpha}=u_{\alpha}^{0}+\varepsilon u_{\alpha}^{(1)}+\varepsilon^{2} u_{\alpha}^{(2)}+\cdots$ and $\phi=\varepsilon \phi^{(1)}+\varepsilon^{2} \phi^{(2)}+\cdots$. The propose is obtained kdv equation. The continuity and momentum Poisson Equations (1)-(3) are treated by perturbed quantities for $n_{\alpha}, u_{\alpha}$, and $\phi$ in terms of $\varepsilon$ and $\varepsilon^{2}$. The first perturbed density and velocity calculations can be expressed as follow:

$$
\begin{gathered}
n_{\alpha 1}=n_{\alpha 0} \eta_{\alpha 0} \phi_{1}, \\
u_{\alpha 1}=\left(v-u_{\alpha 0}\right) \eta_{\alpha 0} \phi_{1} .
\end{gathered}
$$

where, $\eta_{\alpha 0}=M_{\alpha} Q_{\alpha} /\left(v-u_{\alpha 0}\right)^{2}+M_{\alpha} \sigma_{\alpha}$. such that $v \neq u_{\alpha 0} \pm \sqrt{M_{\alpha} \sigma_{\alpha}}$ and that $v \neq>u_{\alpha 0}$ Thus, the signs of $\eta_{\alpha 0}, u_{\alpha 1}$ and $n_{\alpha 1}$ are always similar to $Q_{\alpha}$. The neurtal condition of four densities $n_{p r^{+} 1}, n_{p 1}, n_{p r-1}$ and $n_{e 1}$ are given by:

$$
n_{p r^{+} 1}+n_{p 1}=n_{p r^{-} 1}+n_{e 1} \text {. }
$$

To obtain the $k d v$ equation, the coefficient of $\partial \phi_{2} / \partial \xi$ must be vanished 
which appears in Poisson equation. Thus, the coefficient of $\partial \phi_{2} / \partial \xi$ gives the equation $h_{p r^{+} 0}-h_{p r^{-} 0}+h_{p 0}-h_{e 0}=0$ where, $h_{\alpha 0}=n_{\alpha 0} \eta_{\alpha}$, to find the phase velocity we solve the expressed equation of

$$
\begin{aligned}
& \frac{n_{p r^{+} 0} M_{p r^{+} 0} Q_{p r^{+} 0}}{\left(v-u_{p r^{+} 0}\right)^{2}+M_{p r^{+} 0} \sigma_{p r^{+} 0}}+\frac{n_{p 0} M_{p} Q_{p}}{\left(v-u_{p 0}\right)^{2}+M_{p} \sigma_{p}} \\
& -\frac{n_{p r^{-} 0} M_{p r^{-}} Q_{p r^{-}}}{\left(v-u_{p r^{-} 0}\right)^{2}+M_{p r^{-}} \sigma_{p r^{-}}}-\frac{n_{e 0} M_{e} Q_{e}}{\left(v-u_{e 0}\right)^{2}+M_{e} \sigma_{e}}=0 .
\end{aligned}
$$

which is transformed into the algebraic equation of degree six where $v \neq u_{p r^{+} 0} \pm \sqrt{M_{p r^{+}} \sigma_{p r^{+}}}, u_{p r^{-} 0} \pm \sqrt{M_{p r^{-}} \sigma_{p r^{-}}}, u_{p 0} \pm \sqrt{M_{p} \sigma_{p}}$, and $u_{e 0} \pm \sqrt{M_{e} \sigma_{e}}$. Then, the solution of Poisson's equation can be expressed as:

$$
\frac{\mathrm{d} \phi^{(1)}}{\mathrm{d} \tau}+B \phi^{(1)} \frac{\mathrm{d} \phi^{(1)}}{\mathrm{d} \xi}+A \frac{\mathrm{d} \phi^{(1)}}{\mathrm{d} \xi^{3}}=0 .
$$

where

$$
\begin{gathered}
A=\frac{2}{h_{p r^{+} 1}-h_{p r^{-1}}+h_{p 1}-h_{e 1}}, B=h_{p r^{+} 2}-h_{p r^{-2} 2}+h_{p 2}-h_{e 2}, \\
h_{\alpha 1}=\frac{2 v n_{\alpha 0} M_{\alpha} Q_{\alpha 0}\left(v-u_{\alpha 0}\right)}{\left[\left(v-u_{\alpha 0}\right)^{2}-M_{\alpha} \sigma_{\alpha}\right]^{2}}, h_{\alpha 2}=\frac{n_{\alpha 0} M_{\alpha}^{2} Q_{\alpha}^{2}\left[3\left(v-u_{\alpha 0}\right)^{2}-M_{\alpha} \sigma_{\alpha}\right]}{2\left[\left(v-u_{\alpha 0}\right)^{2}-M_{\alpha} \sigma_{\alpha}\right]^{3}}
\end{gathered}
$$

The solution of kdv is given by for $\phi_{1}, u_{\alpha 1}$ and $n_{\alpha 1}$ are expressed as follow:

$$
\begin{gathered}
\phi_{1}=\phi_{0} \operatorname{sech}^{2}(\gamma Y), \\
u_{\alpha 1}=\left(v-u_{\alpha 0}\right) \eta_{\alpha 0} \phi_{0} \operatorname{sech}^{2}(\gamma Y), \\
n_{\alpha 1}=n_{\alpha 0} \eta_{\alpha 0} \phi_{0} \operatorname{sech}^{2}(\gamma Y) .
\end{gathered}
$$

where, $\phi_{0}=3 v / B, \gamma=\sqrt{v / 4 A}$ and $Y=\xi-v \tau$. Thus. The energy of the plasma is given by:

$$
E_{\text {Total }}=\int_{0}^{Y_{\text {rest }}} E_{\text {Plasma }}(Y ; \lambda) \mathrm{d} Y=\frac{1}{2} \sum_{\alpha} n_{\alpha} u_{\alpha}^{2}+\frac{1}{2} \sum_{\alpha} Q_{\alpha} n_{\alpha} \phi
$$

where, $n_{\alpha} \approx n_{\alpha 0}+\varepsilon n_{\alpha 1}, u_{\alpha} \approx u_{\alpha 0}+\varepsilon u_{\alpha 1}$ and $\phi \approx \varepsilon \phi_{1}$. The total plasma energy of zero and first order perturbations calculated by

$$
E_{\text {Plasma energy }}(Y ; \lambda) \approx \frac{1}{2} \sum_{\alpha}\left(n_{\alpha 0} u_{\alpha 0}^{2}+n_{\alpha 1} u_{\alpha 0}^{2}+2 n_{\alpha 0} u_{\alpha 0} u_{\alpha 1}+Q_{\alpha} n_{\alpha} \phi_{1}\right) .
$$

\section{Renormalization of Plasma Energy Calculation}

Applying to our example injected proton, antiproton, electron, and positron inside the cavity of palladium crystal. The ion Bohm acoustic velocity $C_{s}=u_{e}=u_{p}=1000 \mathrm{~m} / \mathrm{s}$, the density of one mole of electron, positron, proton and anti-proton are $n_{e}=n_{p}=n_{p r^{+}}=n_{p r^{-}}=6.6022 \times 10^{23} \mathrm{~m}^{-3}$, The temperature ratio of electron, positron and proton respectively $\sigma_{e}=\sigma_{p}=1$ and $\sigma_{p r+}=0$, in case of free space, the plasma frequency of antiproton $=4.04 \times 10^{6} \mathrm{rad} / \mathrm{s}$ and 
in case of palladium crystal where, $\varepsilon=13.7 \varepsilon_{0}, \omega_{p r-}=8.2 \times 10^{5} \mathrm{rad} / \mathrm{s} . \quad Y=\xi-v \tau$ will be expressed in renormalized coordinate as $Y=\left(\omega_{p r} / C_{s}\right) x-v(v+1) \omega_{p r} t$ where, $\lambda_{D}=\frac{C_{s}}{\omega_{p r}}, \tau_{D}=1 / \omega_{p r}$.

\section{Results and Discussion}

Our objectives in this section are twofold: first to discuss the solution of the dispersion relation (8) deduced in Section 2, and second to discuss the solution of the kdv Equation (11) derived in the same section. The models of protons-antiprotons-electrons-positrons are studied for unit charge $e=1$ and relative mass $\Lambda=1836$. The unperturbed densities are set to be

$n_{p r^{-} 0}=n_{p r^{+} 0}=n_{e 0}=n_{p 0}=1$, whilest the unperturbed velocities are chosen to be $u_{p r^{+} 0}=0$, and $u_{e 0}=u_{p 0}=1, \sigma_{p r^{+}}=0$, and $\sigma_{p}=\sigma_{e}=1$.

\subsection{Results of Linear Analysis}

With respect to the analysis of the dispersion relation (8) for electrons, protons, positrons and anti-protons plasma, the wave number $k$ is rewritten in terms of $\omega$ to obtain the algebraic equation of an eight degree in $k$. In our solution, we select the positive root that indicates the existence of ion-acoustic waves in the system and the possibility of existing soliton waves. The wave number is plotted against frequency to describe the behavior of the dispersion relation. In Figure 1, the dispersion relation has only one positive root at $\omega=43.8 \mathrm{k}$, which is independent of the parameters $u_{p r^{-} 0}, \sigma_{p r^{-}}$and $\Lambda$. In this regard, the linear dispersion relation is plotted linearly by $\mathrm{k}$ against $\omega$. Over $\omega$ domain from zero to 30,000 as shown in Figure 1. Hence, the reciprocal of the slope is the phase velocity and is equal to 43.8485 .

\subsection{Results of Nonlinear Analyses}

In the present section we display the calculations of the potential and kinetic energies for nonlinear analyses. In Figure 2, the potential $\phi_{1}$ (14) are plotted against the stretched coordinate $Y$ for certain values of the plasma parameters and for the following choices of the unperturbed velocity of antiproton,

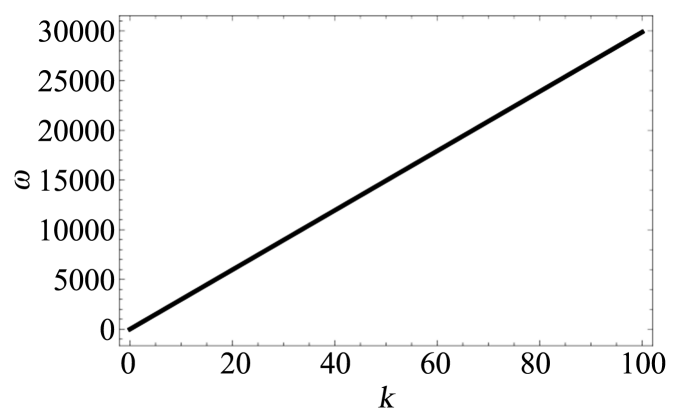

Figure 1. Relation between the wave number $k$ for $n_{p r^{-} 0}=n_{p r^{+} 0}=n_{e 0}=n_{p 0}=1$, $u_{e 0}=u_{p 0}=1, u_{p r^{-}}=0.09, u_{p r^{+}}=0, \sigma_{p r^{+}}=0$, and $\sigma_{p}=\sigma_{e}=1$ for $k$ against $\omega$. 
respectively $u_{p r^{-}}=0.09,0.009$, and 0.0009 . The figures show that the potential is attractive and symmetric about $Y=0$, i.e., Support the possible existence different bound states between the interacting particles. Also, its range is contracted as the unperturbed velocity of antiproton decelerates at low relative velocities.

In Figure 3, the kinetic energy of the plasma corresponding to the zero and first order perturbations are sketched versus the stretched coordinate $Y$ at the
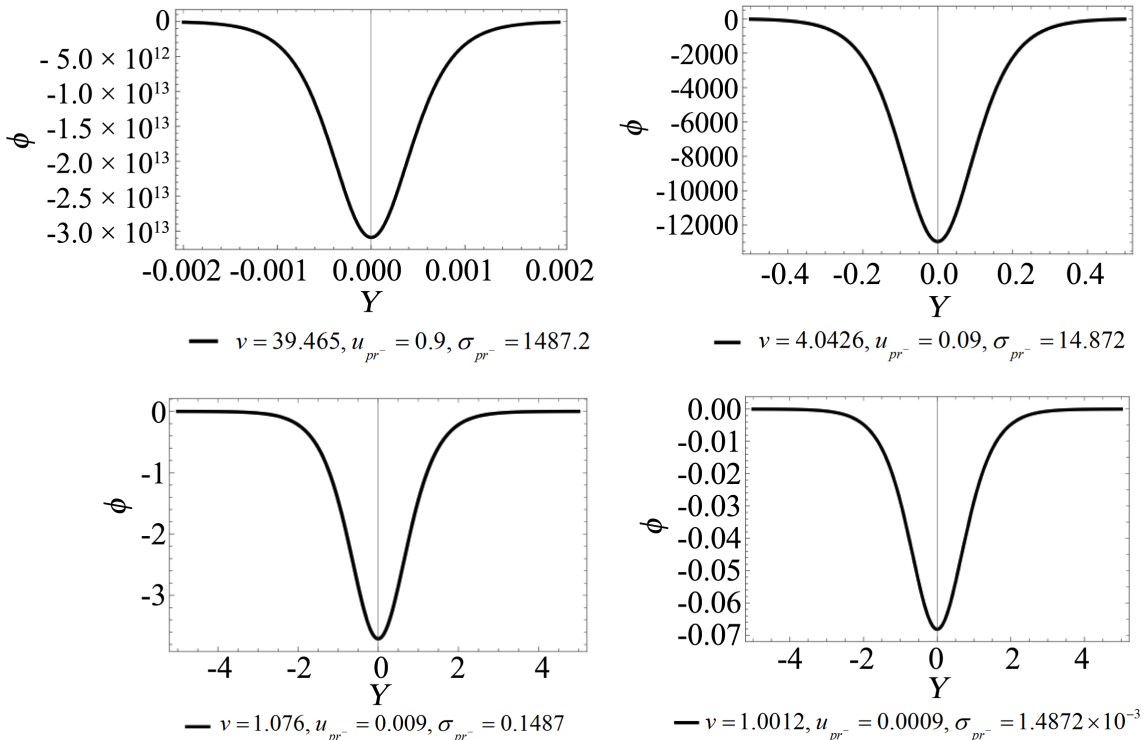

Figure 2. Schematic diagrams for the potential energy calculated using the following parameters $n_{p r^{-} 0}=n_{p r^{+} 0}=n_{e 0}=n_{p 0}=1, u_{e 0}=u_{p 0}=1, u_{p r^{+}}=0, \sigma_{p r^{+}}=0, \sigma_{p}=\sigma_{e}=1$, at the three different values of $u_{p r^{-}}$.
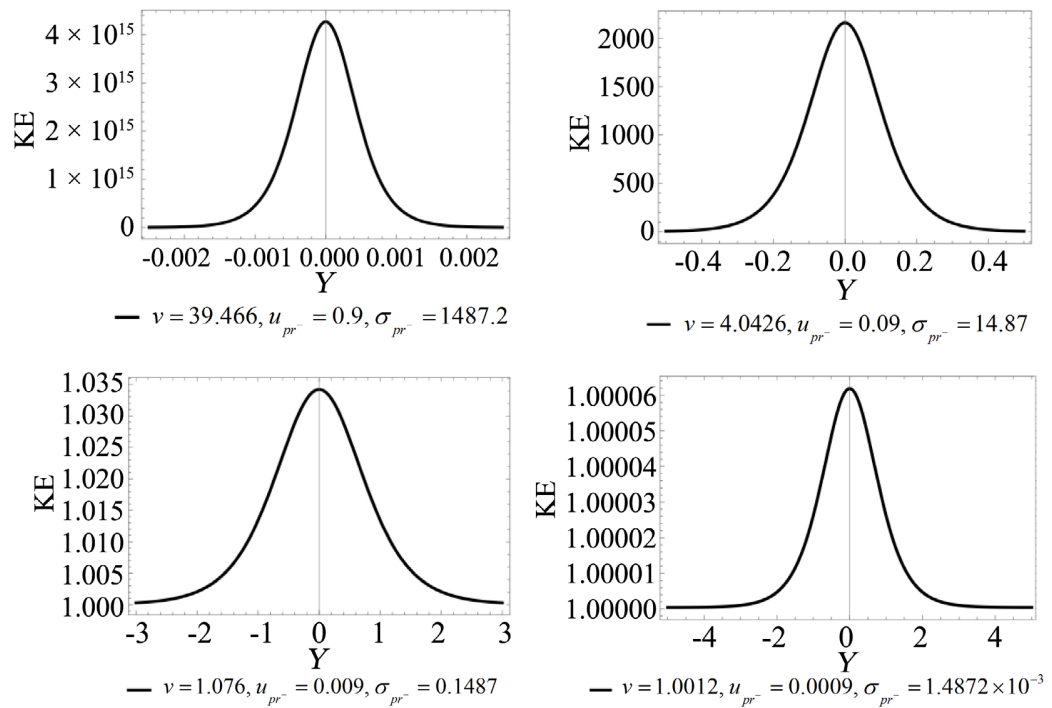

Figure 3. (a)-(c) schematic diagrams for the kinetic energy of our novel system for $n_{p r^{-} 0}=n_{p r^{+} 0}=n_{e 0}=n_{p 0}=1, u_{e 0}=u_{p 0}=1, u_{p r^{+}}=0, \sigma_{p r^{+}}=0, \sigma_{p}=\sigma_{e}=1$, at different values of the antiproton velocities, $u_{p r^{-}}$. 
same parameters used for calculating the potential energy presented in Figure 2. Obviously, the kinetic energy of the plasma increases when the particles are close to each other.

\section{Total Energy Released from Plasma (By Renormalized Our System)}

The ion Debye radius and time of plasma period in case of free space and in palladium crystal respectively $\left.\lambda_{D}\right|_{\varepsilon_{0}}=3.289 \times 10^{-4} \mathrm{~m},\left.\tau\right|_{\varepsilon_{0}}=3.289 \times 10^{-7} \mathrm{~s}$, $\left.\lambda_{D}\right|_{\varepsilon}=1.2175 \times 10^{-3} \mathrm{~m}$, and $\left.\tau\right|_{\varepsilon}=1.2175 \times 10^{-6} \mathrm{~s} . K E(\mathrm{~J} / \mathrm{mole})$ are calculate at $\lambda_{D}=0, t=0,(K E(0,0))$ and at $\left.\lambda_{D}\right|_{\varepsilon_{0}},\left.\lambda_{D}\right|_{\varepsilon}$ and $\left.\tau\right|_{\varepsilon_{0}},\left.\tau\right|_{\varepsilon}\left(K E\left(\lambda_{D}, t\right)\right)$. The energy calculates at free space and in palladium crystal where $\varepsilon=\varepsilon_{0}, 13.7 \varepsilon_{0}$. The energy calculations at our macroscopic study are similar.

\begin{tabular}{ccccc}
\hline$n_{p r^{-}}(\mathrm{m} / \mathrm{s})$ & $V$ & $\sigma_{p r^{-}}$ & $\begin{array}{c}K E(0,0) \\
(\mathrm{J} / \mathrm{mole})\end{array}$ & $\begin{array}{c}K E\left(\lambda_{D}, t\right) \\
(\mathrm{J} / \mathrm{mole})\end{array}$ \\
\hline 900 & $39: 46549227711354$ & 1487.2 & $1.15723 \times 10^{20}$ & $408: 496$ \\
90 & $4: 0426747900049795$ & 14.872 & $8.3114 \times 10^{6}$ & $4: 62805$ \\
9 & $1: 076316038977318$ & 0.14872 & 37.3136 & $10: 1747$ \\
0.9 & $1: 001193627473321$ & $1.487 \times 10^{-3}$ & $0: 610901$ & $0: 574815$ \\
0.09 & $1: 0000524390091419$ & $1.4872 \times 10^{-5}$ & $0: 54891$ & $0: 548721$ \\
\hline
\end{tabular}

In our novel system we could evaluate the plasma energy released of electron, positron, proton and antiproton annihilation in case of free space and in the cavity of palladium crystal. The results show that the time of existing plasma before annihilation around 13.7 time the free space. The amount of energy in case of macroscopic study of the plasma energy is the same in case of free space and palladium crystal. By, changing the velocity of antiproton up to the ion Bohm acoustic wave we could control the amount of energy released from the system. At high speed, the energy of the plasma is very high and within time scale $0.11 \mathrm{~s}$ the amount of energy decay very fast. At very low velocity the energy very low but the system is more stable.

\section{Total Energy Released from the Proposed Annihilates Mechanism}

As we know the electron-positron pair annihilates into two gamma photons the total energy of which is $1.022 \mathrm{MeV}$. Also, the protonium annihilation yields energy of the order of $2 \times 0.938$, i.e. $1.876 \mathrm{GeV}$. Thus, it is anticipated that the yield of the hydrogen-antihydrogen system is $1886.22 \mathrm{MeV}\left(=3.02205748 \times 10^{-13}\right.$ Joule). Which means that we have $3.02205748 \times 10^{-13} \times 6.02214 \times 10^{23}=$ $18.1992532386 \times 10^{10}$ Joule $/$ mole $=1.882 \times 10^{16}$ Joule $/ \mathrm{kg}$. Comparing this result with yields of chemical reaction, nuclear fission and nuclear fusion which are of the order of $1 \times 10^{7}, 8 \times 10^{12}, 1 \times 10^{14} \mathrm{Joule} / \mathrm{kg}$, respectively, we not that our suggested experiment is superior. 


\section{Conclusion}

The one-dimensional hydrodynamic model of linear and nonlinear analyses in the term of electron-positron and proton-antiproton are studied. The linear analyses show a positive root that indicates ion-acoustic waves in the system and the possibility of existing soliton waves. Also, we obtain the calculations of the nonlinear analysis of the deformed $\mathrm{kdv}$ equation and the energy released of the formed plasma. The results show that the lifetime of formed plasma increases inside the palladium crystal 13.7 times concerning the free space. We have the same energy of formed plasma in the case of free space and palladium crystal. The renormalized example study assumes that when the velocity of the antiproton is around the ion-acoustic wave the released energy of the plasma could be reached to $1.15723 \times 10^{20} \mathrm{~J} / \mathrm{mole}$. On the other hand, if the velocity of the anti-proton decreases, the total energy of the plasma system decreases. This result supports very much the conclusion that we suggested model for obtaining huge amount of energy by constructing a plasma state between thermalized hydrogen and antihydrogen in molecular crystals with approximate velocity less than the velocity of ion acoustic wave of the system. This energy could find interesting application in cold fusion and building up engines for space shuttles.

\section{Conflicts of Interest}

The authors declare no conflicts of interest regarding the publication of this paper.

\section{References}

[1] Amoretti, M.E.A., et al. (2002) Production and Detection of Cold Antihydrogen Atoms. Nature, 419, 456-459. https://doi.org/10.1038/nature01096

[2] Bonomi, G., et al. (2005) Antihydrogen Production Mechanisms in ATHENA. Nucl. Phys. A, 752, 97-100. https://doi.org/10.1016/j.nuclphysa.2005.02.046

[3] Abdel-Raouf, M.A. (2019) Matter-Antimatter Physics at Low Energy. Journal of Physics: Conference Series, 1253, Article ID: 012006. https://doi.org/10.1088/1742-6596/1253/1/012006

[4] Abdel-Raouf, M.A. (2020) On Possible Formation of Matter-Antimatter Exotic Molecular Structures. Open Access Library Journal, 7, 1-27.

[5] Abdel-Raouf, M.A. (2020) Novel Consequences of Coexistence of Matter and Antimatter in Nature. Journal of High Energy Physics, Gravitation and Cosmology, 6, 251-258. https://doi.org/10.4236/jhepgc.2020.62019

[6] Abdel-Raouf, M.A. (1989) Possibility of Producing an Intensive Beam of Antihydrogens and Its Consequences. 12 th International Conference on Few Body Problems in Physics, Vancouver, 2-8 July 1989, A17.

[7] El-Tantawy, S.A., Elgendy, A.T. and Ismail, S. (2017) Cylindrical Freak Waves in a Non-Maxwellian Dusty Bulk-Sheath Plasma: An Approximate Solution for the Cylindrical Nonlinear Schrödinger Equation. Physics Letters A, 381, 3465-3471. https://doi.org/10.1016/j.physleta.2017.08.054

[8] El-Tantawy, S.A., Ali Shan, S., Akhtar, N. and Elgendy, A.T. (2018) Impact of Electron Trapping in Degenerate Quantum Plasma on the Ion-Acoustic Breathers and Super Freak Waves. Chaos, Solitons \& Fractals, 113, 356-364. 
https://doi.org/10.1016/j.chaos.2018.04.037

[9] Elgendy, A.T. (2019) Plasma Boundary of Nonlinear Sheath Dynamics for Arbitrary Waveforms in Capacitive Discharge. Journal of Physics: Conference Series, 1253, Article ID: 012010. https://doi.org/10.1088/1742-6596/1253/1/012010

[10] Kouser, S., Shah, K.H., Qureshi, M.N.S. and Shah, H.A. (2020) Nonlinear IonAcoustic Waves in e-p-i Plasmas with $(r, q)$ Distributed Electrons and Positrons. AIP Advances, 10, Article ID: 55123. https://doi.org/10.1063/5.0011128

[11] Malik, H.K., Singh, S. and Dahiya, R.P. (1994) Kadomtsev-Petviashvili Solitons in Inhomogeneous Plasmas with Finite Temperature Drifting Ions. Physics Letters A, 195, 369-372. https://doi.org/10.1016/0375-9601(94)90044-2

[12] Singh, S. and Honzawa, T. (1993) Kadomtsev-Petviashivili Equation for an IonAcoustic Soliton in a Collisionless Weakly Relativistic Plasma with Finite Ion Temperature. Physics of Fluids B: Plasma Physics, 5, 2093-2097.

https://doi.org/10.1063/1.860745

[13] Petrov, A.E., Sarksyan, K.A., Skvortsova, N.N. and Kharchev, N.K. (2001) Observation of Nonlinear Coupling between Drift and Ion-Acoustic Oscillations in LowFrequency Plasma Turbulence. Plasma Physics Reports, 27, 56-61.

https://doi.org/10.1134/1.1339484

[14] Nakamura, Y. (1982) Experiments on Ion-Acoustic Solitons in Plasmas Invited Review Article. IEEE Transactions on Plasma Science, 10, 180-195. https://doi.org/10.1109/TPS.1982.4316165

[15] Lonngren, K.E. (1998) Ion Acoustic Soliton Experiments in a Plasma. Optical and Quantum Electronics, 30, 615-630. https://doi.org/10.1023/A:1006910004292

[16] Myers, S. and Picasso, E. (1990) The Design, Construction and Commissioning of the CERN Large Electron-Positron Collider. Contemporary Physics, 31, 387-403. https://doi.org/10.1080/00107519008213789

[17] Myers, S. (2013) The Large Hadron Collider 2008-2013. International Journal of Modern Physics A, 28, Article ID: 1330035. https://doi.org/10.1142/S0217751X13300354

[18] Georgin, M.P., Jorns, B.A. and Gallimore, A.D. (2018) Experimental Evidence for Ion Acoustic Solitons in the Plume of a Hollow Cathode. 2nd Space Propulsion Conference, Sevilla, 14-18 May 2018, 12 p. 


\section{Appendix A}

In this Appendix we present a brief account on the properties of palladium crystals. The palladium could absorb more than 900 times its own volume of hydrogen and antihydrogen. It expands and becomes harder, stronger, and less ductile in the process. Palladium is a metal in group VIII of the periodic table with atomic number 46 , an atomic weight of 106.4 , and a density of $12 \mathrm{Mg} / \mathrm{m}^{3}$. Its melting point is $1552 \mathrm{C}$, and it boils at $3980 \mathrm{C}$. The electronic configuration of is $(\mathrm{Kr})$ ( $4 \mathrm{~d} 10)(5 \mathrm{~s} 0)$. Its atomic radius is $0.137 \mathrm{~nm}$. At room temperature, Palladium has a face-centered cubic crystal structure with $a=0.388 \mathrm{~nm}$. The linear coefficient of thermal expansion is $12.29 \mathrm{~m} / \mathrm{m} / \mathrm{K}$ and the thermal conductivity is 0.718 $\mathrm{W} / \mathrm{cm} / \mathrm{K}$.

\section{Appendix B}

In the Appendix, we present closed forms of the final analyses of the terms $A_{1}$, $B_{1}$ and $C_{1}$ appeared in Section 2.

$$
\begin{aligned}
& A_{1}=\frac{2 \lambda n_{p r^{+} 0} Q_{p r^{+}} M_{p r^{+}}\left(\lambda-u_{p r^{+} 0}\right)}{\left(\left(\lambda-u_{p r^{+} 0}\right)^{2}-M_{p r^{+}} \sigma_{p r^{+}}\right)^{2}}+\frac{2 \lambda n_{p 0} Q_{p} M_{p}\left(\lambda-u_{p 0}\right)}{\left(\left(\lambda-u_{p 0}\right)^{2}-M_{p} \sigma_{p}\right)^{2}} \\
& -\frac{2 \lambda n_{p r^{-} 0} Q_{p r^{-}} M_{p r^{-}}\left(\lambda-u_{p r^{-} 0}\right)}{\left(\left(\lambda-u_{p r^{-} 0}\right)^{2}-M_{p r^{-}} \sigma_{p r^{-}}\right)^{2}}-\frac{2 \lambda n_{e 0} Q_{e} M_{e}\left(\lambda-u_{e 0}\right)}{\left(\left(\lambda-u_{e 0}\right)^{2}-M_{e} \sigma_{e}\right)^{2}} \\
& B_{1}=\frac{e^{2} n_{0}}{2}\left[\frac{3\left(\lambda-u_{p r^{+} 0}\right)^{2}-\sigma_{p r^{+}}}{\left(\left(\lambda-u_{p r^{+} 0}\right)^{2}-\sigma_{p r^{+}}\right)^{3}}+\frac{(1836)^{2}\left(3\left(\lambda-u_{p 0}\right)^{2}-1836 \sigma_{p}\right)}{\left(\left(\lambda-u_{p 0}\right)^{2}-\sigma_{p}\right)^{3}}\right. \\
& \left.-\frac{3\left(\lambda-u_{p r^{-} 0}\right)-\sigma_{p r^{-}}}{\left(\left(\lambda-u_{p r^{-} 0}\right)^{2}-\sigma_{p r^{-}}\right)^{3}}-\frac{(1836)^{2}\left(3\left(\lambda-u_{e 0}\right)^{2}-1836\right)}{\left(\left(\lambda-u_{e 0}\right)^{2}-1836\right)^{3}}\right] \\
& C_{1}=h_{p r^{+} 0}+h_{p 0}-h_{p r^{-} 0}-h_{e 0} \\
& =\frac{Q_{p r^{+}} M_{p r^{+}} n_{p r^{+} 0}}{\left(\lambda-u_{p r^{+} 0}\right)^{2}-\sigma_{p r^{+}}}+\frac{Q_{p} M_{p} n_{p 0}}{\left(\lambda-u_{p 0}\right)^{2}-\sigma_{p}} \\
& -\frac{Q_{p r^{-}} M_{p r^{-}} n_{p r^{-} 0}}{\left(\lambda-u_{p r^{-} 0}\right)^{2}-\sigma_{p r^{-}}}-\frac{Q_{e} M_{e} n_{e 0}}{\left(\lambda-u_{e 0}\right)^{2}-M_{e} \sigma_{e}}
\end{aligned}
$$

